\title{
Expressão da p53 no Tumor e no Epitélio Oral em Pacientes com Câncer de Boca e Faringe
}

\section{Expression of p53 in the Tumor and Oral Epithelium in Patients with Cancer of Mouth and Pharynx}

\author{
Fabiane Dittrich Santos*, Jair Montovani**, Cleverson Teixeira Soares***, Lídia Raquel de Carvalbo****. \\ * Mestre em Cirurgia pela Faculdade de Medicina de Botucatu. Cirurgiã Dentista \\ ** Livre-Docente em Otorrinolaringologia. Coordenador de Disciplina em PG sensus stricto. \\ **** Doutorado. Doutor em Patologia do Hospital de Base de Bauru - SP. \\ **** Doutorado. Professora Doutora do Instituto de Biociências de Botucatu - Unesp. \\ Instituição: Faculdade de Medicina de Botucatu - Depto. OFT/ORL/CCP \\ Botucatu / SP - Brasil. \\ Endereço para correspondência: Jair Cortez Montovani - Faculdade de Medicina de Botucatu - Depto. OFT/ORL/CCP - Distrito de Rubião Júnior s/n - Botucatu / SP \\ - Brasil - CEP: 18618-970 - Telefax: (+55 14) 3811-6256/6081 - E-mail: montovan@fmb.unesp.br \\ Artigo recebido em $1^{\circ}$ de Setembro de 2010. Artigo aprovado em $1^{\circ}$ de Outubro de 2010.
}

\section{RESUMO}

Introdução:

Objetivo:

Método:

Resultados:

Conclusão:

Palavras-chave:

\section{SUMMARY}

Introduction:

Objective:

Method:

Results:

Conclusion:

Keywords:
Expressiva porcentagem de pacientes com carcinomas de boca e faringe apresentam superexpressão da proteína p53 induzida por tabaco, álcool e radioterapia.

Descrever a expressão da p53 em áreas de mucosa normal adjacente ao tumor e em carcinomas da boca e faringe.

Estudo prospectivo, com seguimento clínico por um ano, de 24 pacientes com câncer espinocelular de boca e faringe. Foram feitas biópsias na neoplasia e em áreas de mucosa normal adjacente ao tumor, antes e 9 meses após a radioterapia, e realizado estudo imunohistoquímico da expressão da p53.

Antes da radioterapia, houve alteração da expressão da p53 em 20 das 24 biópsias feitas na neoplasia e em 14 nas de mucosa normal adjacente ao tumor. Onze paciente morreram antes de 1 ano de seguimento clínico. Dos 2 pacientes iniciais com aumento da p53 após a radioterapia continuava aumentada em 7 na área da neoplasia e em 6 nas áreas de mucosa normal. Observou-se associação da p53 com o tabagismo e estádio do tumor ( $<$ < 5\%) mas não com o grau de diferenciação celular e alcoolismo.

O aumento da expressão da p53 foi observado tanto na área da neoplasia como em mucosa normal na maioria dos pacientes com carcinoma de boca e faringe antes e após a radioterapia. Houve correlação estatisticamente significante da expressão da p53 com o tabagismo e estádio da neoplasia. neoplasias bucais, neoplasias faríngeas, genes p53, cocarcinogênese.

Expressive percent of patients with oral and oral pharynx carcinomas presents with overexpression of protein $\mathrm{p} 53$ induced by tobacco, alcohol and radiotherapy.

To describe the p53 expression in areas of the normal mucosa adjacent to the tumor and in mouth and pharynx carcinomas.

Prospective study with clinical follow-up of one year of 24 patients with oral and oral pharynx spinocellular carcinoma. We performed biopsies in the neoplasm and areas of the normal mucosa adjacent to the tumor, before and 9 months following radiotherapy and an immunohistochemical study of p53 expression.

Before radiotherapy, there was a change to the p53 expression in 20 out of the 24 biopsies made in the neoplasm and in 14 in those of normal mucosa adjacent to the tumor. Eleven patients died 1 year before clinical follow-up. From the 2 initial patients with increase of p53 after radiotherapy it remained increased in 7 in the neoplasm region and in 6 in the normal mucosa regions. We noticed association of $\mathrm{p} 53$ with smoking and tumor stage $(\mathrm{p}<5 \%)$, but not with the degree of cellular differentiation and alcoholism.

The increase to the p53 expression was viewed both in the neoplasm region and in the normal mucosa in most patients with oral and oral pharynx carcinoma before and after radiotherapy. There was a statistically significant correlation of the p53 expression with smoking and neoplasm stage. mouth neoplasms, pharynx neoplasms, p53 genes, cocarcinogenesis. 


\section{INTRODUÇÃO}

O câncer de cabeça e pescoço (CCP) tem uma incidência de dois a três por cento de todas as neoplasias humanas e ocupa a sexta posição como o câncer mais frequente em todo o mundo. Tipicamente ocorre mais em pacientes do sexo masculino, entre a quinta e oitava década de vida, sendo menos comum em pacientes com menos de 45 anos de idade. Quarenta por cento do câncer de cabeça e pescoço se localizam na cavidade oral, 25\% na laringe, $15 \%$ na faringe e $20 \%$ ocorrem em outros sítios, incluindo as glândulas salivares. OCCP representa importante problema de saúde, sendo responsável por quase 200.000 mortes anualmente em todo o mundo. Na Índia, o carcinoma espinocelular da boca (CEC) corresponde, aproximadamente, a 50\% dos novos casos de cânceres diagnosticados e tem sua incidência crescente, apesar dos avanços na prevenção e em novas terapias $(1,2)$. Porém, as taxas de morbidade e mortalidade tiveram poucas melhorias durante os últimos 30 anos, principalmente quando ele ocorre em pacientes jovens, antes da quarta e quinta décadas de vida $(3,4)$. Observações clínicas recentes mostraram que o câncer oral em pacientes jovens tem uma etiologia e progressão clínica particular, nas quais predominam fatores genéticos independentes de fatores exógenos carcinogênicos, como o tabagismo e o alcolismo $(5,6,7)$.

Quando ocorre a exposição a carcinogenéticos em células como as da boca e faringe, pode haver a "malignização" dessas células, tendo como primeiro momento o aparecimento de células atípicas e displásicas, com diversas alterações genéticas e moleculares, cuja resultante final é uma neoplasia $(7,8)$. Assim, células geneticamente alteradas, gradualmente proliferam-se e se expandem para áreas vizinhas, ainda sem neoplasias, mas já vulneráveis a mutações celulares. Essasáreas, normais ao exame clínico e a macroscopia óptica, têm potencialidade para evoluírem para neoplasias, o que explicaria muitas das situações de recidivas neoplásicas e de ocorrência de um segundo tumor primário $(9,10)$. A probabilidade que isso ocorra em câncer de boca é de 10 a 25\%, principalmente porque muitos desses pacientes mantêm as características anteriores de exposição a agentes carcinogênicos, como o hábito de fumar e de ingestão de bebidas alcoólicas, associadas agora a irradiação terapêutica (11).

Entretanto, ainda hoje, o conceito da evolução de células normais a microscopia óptica para neoplásicas ainda não está bem claro. Um conceito bastante atual é que essas células normais, adjacentes ao tumor, estariam apenas em estágios diferentes do ciclo celular, dormentes, mas já com potenciais mutações nucleares para células neoplásicas $(12,13)$.
Pesquisas biomoleculares indicam que essas células têm determinados genes que sofrem mutações, dependentes ou não da ação de fatores exógenos (14). As alterações dos genes e da expressão das proteínas que eles codificam fariam com que as células perdessem a capacidade de regular o seu crescimento, favorecendo o aparecimento de neoplasias (15).

Um dos primeiros genes mais estudados sob esse ponto de vista é Tp53 e sua expressão, a proteína p53. Quando uma célula é exposta a agentes carcinogênicos, há aumento dos níveis de proteína p53, que se ligam a fatores de transcrição, impedindo que a célula entre na fase $S$ do ciclo celular (16). A parada reversível do ciclo celular na transição C1-S permite a reparação do DNA e o estabelecimento da integridade do genoma. Quando as alterações do DNA excedem a capacidade de reparação, ocorre morte celular por apoptose e, não raro, há perda do controle de crescimento celular. O que se observa são células evolutivas, imaturas com grau acentuado de aneuploidia, perda de heterozigose, expressão de DNA aberrante $(17,18,19,20)$.

Nesse momento, a importância do gene Tp 53 seria a do "guardião do genoma", com capacidade de controlar a qualidade da duplicação do DNA ao detectar um erro na sequência do ciclo celular, provocando a apoptose celular, o que impediria a perda do controle da diferenciação celular $(16,17)$.

Entretanto, não há unanimidade quanto a essas observações. Estudos imunopatológicos de lesões da boca, faringe e laringe, não encontraram alterações estatisticamente discrepantes entre porcentagem alterada da p53, gravidade da displasia epitelial e lesões neoplásicas $(18,19,20,21)$. Uma explicação para esses achados discordantes é que, nem sempre, a super ou falta de expressão da p53 e outros marcadores celulares podem indicar presença ou ausência de neoplasia. Outra hipótese é que a expressão da p53 estaria mascarada pela deleção gênica ou, ainda, estar ligada a vírus, como o hpv $(22,23)$.

Descrição atual interessante é que o estudo de alterações gênicas, como as da p 53, possa ser utilizado para o prognóstico de resistência tumoral a radioterapia e quimioterapia $(24,25,26)$. Ainda hoje, na prática clínica, atribuímos a resistência tumoral ao tratamento às vantagens seletivas das células neoplásicas sobre as normais, pela perda dos mecanismos de controle que regulam o seu crescimento e a proliferação celular $(27,28)$. A aplicabilidade clínica desses conceitos, como por exemplo, a de ter valores preditivos de morbidade e mortalidade de pacientes portadores de câncer de boca e faringe seria um grande avanço $(29,30)$. 
Infelizmente, ainda hoje, a escolha da terapêutica em pacientes com esse tipo de câncer baseia-se, quase sempre, no estadiamento clínico e graduação histológica das neoplasias. Embora conceitos atuais de preservação de órgãos, associados à quimioterapia e radioterapia, até então tidas como coadjuvantes em câncer de cabeça e pescoço, ganharam espaço nas terapêuticas desses tumores, diminuindo a extensão da cirurgia e melhorando a qualidade de vida, ainda não se observa o emprego da biologia molecular como facilitadora para a escolha da modalidade de tratamento.

Sendo assim, o objetivo desse trabalho foi descrever as alterações gênicas, especificamente da expressão da p53, em lesões neoplásicas da mucosa oral e faríngea e nas áreas adjacentes ao tumor, normais a histopatologia, após a radioterapia, em indivíduos com carcinomas espinocelulares de boca e faringe.

\section{MÉTODO}

O presente trabalho foi submetido e aprovado sem restrições pelo Comitê de Ética e Pesquisa de nossa instituição, sob número 435/2002-CEP. O estudo é clínico prospectivo, seguimento de um ano de vinte e quatro pacientes portadores de carcinoma espinocelular da boca e faringe (base da língua). Os critérios de inclusão dos pacientes no estudo foram: indivíduos sem confirmação diagnóstica de câncer, virgens de tratamento, resultado do exame histopatológico da neoplasia feito em nosso hospital, confirmando ser carcinoma espinocelular e da mucosa adjacente ao tumor com ausência de neoplasia. O tratamento escolhido foi cobaltoterapia associados e ou quimioterapia, não tendo os autores principais participado na escolha de tratamento. As variáveis estudadas foram idade, sexo, tabagismo, ingestão de bebidas alcoólicas, classificação TNM do tumor, local do tumor e presença ou ausência da expressão da p53.

Foram incluídos, nesse estudo, 24 pacientes com câncer da boca e faringe (base da língua) com idade variando entre 43 e 95 anos (média 69 anos), sendo 22 $(91,7 \%)$ do sexo masculino e $2(8,3 \%)$ do sexo feminino (Tabela 1).

Quinze pacientes eram tabagistas, mais de um maço/dia, em média há mais 10 anos (positivo), 2 eram não tabagistas (negativo) e 7 tinham abandonado o tabagismo antes da primeira consulta em nosso hospital. Em relação ao uso de álcool, dez pacientes eram etilistas, quatro não eram etilistas (negativos) e dez relataram ter parado de beber e fumar quando foram informados que estavam ou poderiam estar com câncer. Estavam, em média, há 4 meses sem ingerir bebida alcoólica ou fumar.
Tabela I . Resultados das lâminas quantificando a expressão da proteína p53 antes e após a radioterapia.

\begin{tabular}{|c|c|c|c|c|c|}
\hline \multirow[t]{2}{*}{$\mathrm{N}^{\mathrm{O}}$} & \multirow[t]{2}{*}{ ID } & \multicolumn{2}{|c|}{ Pré-radioterapia } & \multicolumn{2}{|c|}{ Pós-radioterapia } \\
\hline & & MNA & T & MNA & T \\
\hline | & 43 & $08+91-$ & $88+$ & & \\
\hline 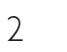 & 69 & $87+13$ & & $M 66+34-$ & $M 54+4$ \\
\hline 3 & 77 & $35+65-$ & $69+31-$ & Óbito & \\
\hline 4 & 46 & $02+98$ & $70+30$ & pito & \\
\hline & 65 & $16+84$ & $74+26-$ & +84- & $M 45+5$ \\
\hline 6 & 71 & $14+86-$ & $0+100$ & M23 + 77- & $\mathrm{M4}+96$ \\
\hline , & 62 & $2+98-$ & $58+42-$ & jito & \\
\hline 8 & 58 & $17+83-$ & $36+64-$ & $M 29+48$ & $M 40+6$ \\
\hline 9 & 95 & $7+93-$ & $94+6-$ & +56 & $M 45+6$ \\
\hline 10 & 48 & $14+86$ & $0+100$ & bito & \\
\hline | | & 51 & $64+36-$ & $69+31-$ & $M 22+78$ & $M 26+7$ \\
\hline 12 & 51 & $6+94-$ & $2+98-$ & MIO+90- & $M \mid I+8$ \\
\hline 13 & 56 & $7+/ 93-$ & $18+/ 82-$ & Óbito & \\
\hline 14 & 53 & $25+775$ & $55+/ 45$ & M5 +/95- & $12116+18$ \\
\hline 15 & 49 & $3+/ 97$ & $49+/ 51-$ & $M 42+/ 58$ & $\mathrm{~T} 33+16$ \\
\hline 16 & 52 & $17+/ 83-$ & $77+/ 23-$ & bito & \\
\hline 17 & 89 & $20+/ 80$ & $56+/ 44-$ & bito & \\
\hline 18 & 83 & $3+/ 97-$ & $0+/ 100-$ & $M 2+/ 98-$ & $\mathrm{M} 9+19$ \\
\hline 19 & 47 & $5+/ 95-$ & $74+/ 26-$ & & \\
\hline 0 & 49 & $44+/ 56$ & $95+5$ & $\mathrm{MIO}+/ 90$ & $13+19$ \\
\hline 1 & 76 & $32+168$ & $42+/ 58$ & Óbito & \\
\hline 22 & 68 & $11+/ 89$ & $71+/ 29$ & $\mathrm{MO}+/ 100$ & $M 4+/ 96$ \\
\hline - & 76 & $29+\Pi 71-$ & $61+/ 39$ & Óbito & \\
\hline 24 & 63 & $1+/ 99-$ & $64+/ 36-$ & $M 9+/ 91-$ & $\mathrm{M} 6+/ 9$ \\
\hline
\end{tabular}

Legenda: MNA: Mucosa Normal Adjacente; T: Tumor; Positiva: +; Negativa: - A expressão é positiva quando apresentava 10 ou mais células coradas num total de 100 células.

Quanto à localização da neoplasia, doze pacientes tinham lesão na base de língua (50\%), cinco pacientes assoalho de boca, dois do lado direito e três no esquerdo, seis em pilar amigdaliano ( 3 direito e 1 esquerdo), um em área retromolar. Quatro tinham estádio clínico T1NOM0 (16,7\%), seis pacientes T2NOMO (25\%), sete pacientes T3N2MO (29,2\%) e sete pacientes T4N2MO (29,2\%) (Tabela 1).

Foram feitas duas biópsias iniciais em locais diferentes, sendo a primeira na lesão neoplásica (A1) e a segunda biópsia distando um centímetro, em média, da neoplasia, em mucosa normal ao exame clínico e histopatológico (A2). Após o término do tratamento radioterápico e ou quimioterápico, foram feitas novamente duas novas biópsias, uma na neoplasia ou no local demarcado antes de se iniciar o tratamento e a outra em mucosa normal. O intervalo de tempo entre a primeira e a segunda biópsia foi de 12 meses.

Cada um dos fragmentos teciduais foi fixado em formalina, incluído em parafina separadamente e colorido pela técnica de hematoxilina-eosina. Foi realizada a análise 
histológica e a graduação histológica: pouco, moderadamente ou bem diferenciado. Quanto ao grau de diferenciação histológica encontramos 8 indivíduos com carcinomas moderadamente diferenciados (Figura 1A), 14 bem diferenciados (Figura 1B) e 2 pouco diferenciados .

Os fragmentos das amostras teciduais (A1) e (A2) foram cortados em blocos e a detecção do gene supressor tumoral TP53 foi realizada por meio de reação imunohistoquímica com o anticorpo monoclonal anti-p53 produzido em camundongos (DAKO Cód. M7001).

Os resultados foram avaliados em microscopia óptica comum, sendo a reação positiva representada pela deposição do cromógeno nos sítios de ligação antígeno-anticorpo, conferindo marcação nuclear a células que expressam os antígenos estudados. A análise da expressão foi considerada positiva, à microscopia óptica, quando detectamos o núcleo da célula com forte coloração marrom (Figura 1C) e negativa quando marrom claro ou não coradas (Figura 1D). O critério positivo da expressão da p 53 foi dado pela contagem de 10 ou mais células (10\%), num total de 100 células por retículos, contando-se as células em no mínimo três retículos (Hamilton and AlLan, 1995) (33).

Foi feito estudo comparativo da expressão da p53 com as variáveis localização do tumor, alcoolismo, tabagismo e estadiamento. Para a análise estatística usamos os testes de Qui-quadrado, o exato de Fischer e de McNemar. O nível de significância utilizado foi de $\mathrm{p}<0,05$.

\section{RESULTADOS}

Observamos a superexpressão da proteína p 53 na biópsia inicial, antes do tratamento, em 14 das 24 amostras de mucosa normal adjacente (MNA) e em 20 das vinte e quatro amostras das lesões neoplásicas. Após o tratamento radioterápico e ou quimioterápico, das catorze amostras de MNA, com expressão positiva da p53, 6 mantinham a p53 superexpressa e 2 negativas. Nas 20 amostras de neoplasia com expressão da p53 aumentada, elas se mantinhas positivas em sete pacientes, dois casos apresentaram expressão negativa da p53. Onze pacientes não puderam ser avaliados por irem a óbito antes da segunda avaliação imunohistoquímica.

Dos pacientes que foram a óbito um apresentava estádio T1, dois estádio T2 (18,2\%), seis (54,6\%) estádio T3 e 2 estádio T4 $(18,2 \%)(p<0,05)$. Em relação à graduação histológica desses pacientes, observamos que cinco casos eram de CEC bem diferenciado, cinco, moderadamente diferenciado e um pouco diferenciado. Houve diferença estatisticamente significante para a relação da superexpressão da p53, tanto no tumor como na mucosa

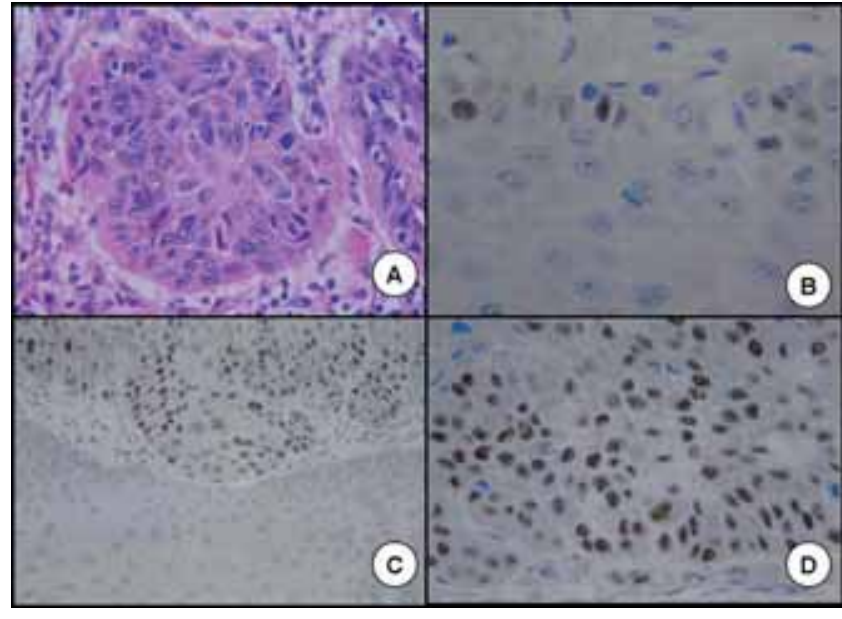

Figura 1. A. Carcinoma espinocelular moderadamente diferenciado de faringe. B. Carcinoma espinocelular bem diferenciado de boca. C. Expressão positiva da p53 em carcinoma espinocelular de boca. D. Expressão negativa da p53 em carcinoma espinocelular de boca.

Tabela 2. Estadiamento dos pacientes e expressão dap 53 na neoplasia antes e após tratamento radioterápico.

\begin{tabular}{lcccc}
\hline Estadiamento & \multicolumn{5}{c}{ Expressão da p53 } \\
(TNM) & Antes daradioterapia & Apósaradioterapia \\
& N & P & N & P \\
\hline T1 & 1 & 3 & 2 & 0 \\
T2 & 0 & 6 & 0 & 2 \\
T3 & 1 & 6 & 0 & 3 \\
T4 & 2 & 5 & 0 & 2 \\
\hline Total & 4 & 20 & 2 & 7 \\
\hline
\end{tabular}

Legenda: N: negativa; P: positiva *Após um ano de seguimento clínico apenas 13 pacientes continuaram vivos.

Tabela 3. Expressão dap 53 na neoplasia e na mucosa normal adjacente à neoplasia.

\begin{tabular}{lcc}
\hline Graduaçãohistológica & \multicolumn{3}{c}{$\begin{array}{c}\text { Expressão da p53 } \\
\text { Neoplasia }\end{array}$} \\
& N & P \\
\hline BD & 2 & 12 \\
MD & 1 & 7 \\
PD & 1 & 1 \\
\hline Total & 4 & 20
\end{tabular}

Legenda: N: negativa; P: positiva; BD: bem diferenciado; MD: moderadamente diferenciado; PD: Pobremente diferenciado.

normal adjacente, com o estádio do tumor, mas não com grau de diferenciação da neoplasia (Tabelas 2 e 3). Observação importante é que cinco pacientes com superexpressão da p53 tinham idade abaixo de 50 anos. Também a associação expressão positiva da p53 com fatores exógenos foi estatisticamente evidente para o 
tabagismo $(\mathrm{p}=0,005)$ e não significativa para o uso do álcool $(\mathrm{p}=0,22)$.

\section{DISCUSSÃO}

Os dados do nosso trabalho confirmam os da literatura, ou seja, a incidência do carcinoma espinocelular de boca e faringe é predominante em homens, acima da quinta década de vida e tendo como fatores de risco o tabagismo e o etilismo $(1,2)$. Esses trabalhos relatam que isoladamente o fumo aumenta o risco de desenvolvimento de câncer de seis vezes e o álcool 3,5 vezes. Quando associados, esses indicadores de risco podem aumentar em até 15 vezes a possibilidade de desenvolver câncer de boca e faringe, sendo que o potencial de transformação maligna induzida por esses agentes é duradouro e que o risco é dose-dependente $(7,11)$. Em nosso estudo, essas observações não foram diferentes, sendo que $90 \%$ dos pacientes portadores de câncer de boca eram tabagistas e 75 a 80\% eram etilistas, aqui considerando os indivíduos que relataram o abandono do uso de álcool e do tabagismo poucas semanas antes do diagnóstico definitivo do câncer.

Entretanto, a observação de carcinoma espinocelular de boca e faringe em cinco pacientes, abaixo de 50 anos, com características clínicas neoplásicas agressivas, mostranos que o estabelecimento da patogenia do câncer nesses indivíduos poderia estar associada a outros fatores de risco, como a de características genéticas herdadas, e não apenas a de mutações celulares induzidas por agentes exógenos carcinogênicos $(3,4,5,6)$. Nesses casos, o ideal para o diagnóstico e prognóstico de vida, é que deveríamos utilizar cada vez mais métodos que quantifiquem, não só achados histológicos como tipos e graus de diferenciação celular das neoplasias mas também as características moleculares e susceptibilidade genética das células neoplásicas dos carcinomas da boca e faringe $(15,24,25,26)$. Nas duas últimas décadas, as descrições dos genes TP53, K167, entre outros, com funções de controle de crescimento celular, salientaram novas perspectivas quanto ao tratamento e prognóstico desse tipo de câncer (30).

Esses novos conceitos são bastante instigantes para entendermos se a progressão de células normais e ou displásicas para a malignização, principalmente em indivíduos jovens, ocorrem por meio de alterações e mutações genéticas cancerígenas que poderiam já estar presentes e ainda não visíveis à histologia convencional $(27,28,29)$. Esse conceito de que teoria genética do câncer é bastante atual foi o que nos motivou a pesquisar alterações gênicas não só no tumor como também em mucosa normal adjacente à neoplasia (12). Em nossos achados, as expressões aumentadas da p53 ocorreram tanto na área do tumor como na mucosa normal. Para Regesi et al. (1995) (19) e
Aguiar e Araújo (1997) (20) essas alterações podem ter valor de predição quanto ao aparecimento de uma neoplasia ou de resistência à radioterapia e quimioterapia. Nossos dados, apesar de não conclusivos, parecem confirmar essas informações, pois a maioria dos pacientes que apresentaram superexpressão da p53 tinham tumores grandes e responderam mal à radioterapia. Nestes indivíduos, a persistência da superexpressão da $\mathrm{p} 53$, tanto no tumor como na mucosa normal poderia ser um indicativo de uma maior resistência ao tratamento radioterápico e, consequentemente, afetando a sobrevida de pacientes com câncer de boca e faringe $(30,31)$.

Essas observações porém não são conclusivas. A correlação clinicopatológica mostrada por nós, entre tamanho de tumor e superexpressão da p53, indica-nos que possíveis alterações gênicas e moleculares, entre elas da expressão da p53, quando detectadas em indivíduos com carcinomas de boca e faringe, podem ser um fator de mau prognóstico, principalmente quando da expressão da p53 e outras proteínas emáreas de mucosa normais adjacentes ao tumor $(2,20,26)$. Esses achados mostrariam uma possível origem multicêntrica do câncer oral e faríngeo, devendo então o examinador fazer biópsias e exames em outros sítios próximos à lesão e não só na neoplasia, lesões moleculares poderiam estar presentes no epitélio adjacente ao tumor, mas não visíveis à microscopia óptica (12). Isso atende a teoria de malignização de campo (12), explicando o aparecimento de um segundo tumor primário e de muitas recidivas neoplásicas $(9,10,11)$. Enfim, a exata característica molecular da susceptibilidade genética desse campo tecidual alterado não é ainda clara, mas genes supressores de tumor como Tp53 podem ser a chave nos estágios iniciais do câncer, abrindo inúmeras possibilidades de se prever em um futuro próximo resultados melhores de tratamento e prognóstico de vida.

\section{CONCLUSÃO}

Os achados do presente estudo mostraram que a expressão da p 53 estava aumentada em 20 das 24 biópsias feitas na área da neoplasia eem 14 biópsias da mucosa normal adjacente ao tumor antes do início da radioterapia e mantendo-se biópsia após o término do tratamento radioterápico. Houve correlação estatisticamente significante entre aumento da p 53 com o tamanho da neoplasia e tabagismo, mas não com o grau de diferenciação celular.

\section{REFERÊNCIAS BIBLIOGRÁFICAS}

1. Parkin DM, Pisani P, Ferlay J. Estimates of the worldwide incidence of eighteen majors cancers in 1985. Int J Cancer. 1993, 54:594-606. 
2. Kowalski LP, Franco EL, Torloni H, Fava AS, Andrade Sobrino J, Ramos G, Oliveira BV, Curodo MP. Lateness of diagnosis of oral and oropharyngeal carcinoma: factors related to the tumor, the patients and health factors. Oral Oncol Eur J Cancer. 1994, 30:167-73.

3. Kock WM, Lanzo M, Seurel D, Zohura KM, Sidransky. Head and neck cancer in nonsmokers: a distinct clinical and molecular entity. Laryngoscope. 1999, 109:54451.

4. Martin-Granizo R, Rodrigues-Campo F, Naval Z, Gonçalez FJW. Squamous cell carcinoma of the oral cavity in young people- a comprehensive literatura review. Oral Oncol. 2001, 33:401-18.

5. Mackenzie J, Ah-See K, Thakker M, Sloan P, Marau AG, Birch J, et al. Increasing incidence of oral cancer among young person: what is the aethiology? Oral Oncol. 2000, 36:387-9.

6. Schantz SP, Yu G. Head and neck cancer incidence trends in young americans, 1973-1997, with a special analysis for tongue cancer. Arch Otolaringol Head Neck Surg. 2002, 128:268-74

7. Lewellyn CD, Johnson NW, Warnakulasurixa KA. Risk factors for squamous cell carcinoma of the oral cavity in young people-comprehensive: literature review. Oral Oncol. 2001, 37(5):401-18.

8. Rodrigo JP, Suaréz C, González MV, Lazo PS, Ramos S, Coto E, Alvarez I, García LA, MartínezJA. Variation of genetic alterations in different sites of head and neck cancer. Laryngoscope. 2001, 111:1297-01.

9. Jones AS, Phillips DE, Husband D. Second primary tumors in patients with head and neck squamous cell carcinoma. Cancer. 1995, 75:1343-53.

10. Cianfriglia FD, Gregorio DA, Manieri A. Mutiple primary tumor in patient with oral squamous cell carcinoma. Oral Oncol. 1999, 35:157-63.

11. Franco EL, Kowalski LP, Kanda LL. Risk factors for second cancers of the upper respiratory and digestive systems: a case- control study. J Clin Epidemiol. 1991, 44:615-25.

12. CalifanoJ, Vander Riet O, Westra W, NawwrozH, Clyman G, Prantadon S, et al. Genetic progression model for head and neck: implications for field cancerization. Cancer Res. 1996, 56:2488-92.

13. Neville BW, Day TA. Oral cancer and precancerous lesions. Cancer J Clin. 2002, 52:195-215.
14. Nagai MA, Miracca EC, Yamoto L, Kowalski LP, Bremtani RR. TP53 Mutations in upper aerodigestive squamous cell carcinomas from a group of Braziliam patients. Am J Surg. 1995, 170:492-4.

15. Coltrera MD, Zarbo RJ, Sakr WA, Gown AM. Markers for displasya of the upper aero digestive tract. Suprabasal expression of PCNA, p53 e CK 19 in alcohol-Fixed, embebeded tissue. Am J Pathol. 1992, 141:818-25.

16. Veiculoscu NE, El-Deiry WS. Biological and clinical importance of the p53 tumor suppressor gene. Clin Chem. 1996, 42:858-68.

17. Shin DM, Kim J, Ro JY, Hittelman J, Roth JA, Hong WK, Hittelman WN. Activation of $\mathrm{p} 53$ gene expression in premalignant lesions during head and neck tumorigenesis. Cancer Res. 1994, 54:321-6.

18. Wood MW, Medina JE, Thompson GC, Houck JR, Min $\mathrm{KW}$. Accumulation of the $\mathrm{p} 53$, supressor gene product in oral leukoplakia. Otolaryngol Head Neck Surg. 1994, 111:758-763.

19. Regesi JA, Zarbo RJ, Regevi E, Pysanty S, Silver-Man S, Gazit D. p53, protein expression sequential biopsies of oral displasias and in situ carcinomas. J Oral Pathol Med. 1995, 24:18-22.

20. Aguiar MCF, Araújo VC. p53 protein expression in lining epithelium adjacent to oral squamous cell carcinoma. Rev Pos Grad. 1997, 4:14-9.

21. Chung KY, Mukhopadhyay T, Kim J, Casson A, Ro JY, Goepfert H, Hong WK, Roth JA. Discordant p53 gene mutations in primary head and neck cancers and corresponding second primary cancers of the upper aerodigestive tract. Cancer Res. 1993, 53:1676-83.

22. Naggar AKE, Lai S, Glayman GL, Mims B, Lippman SM, Coombes M, Luna MA, Lozano G. p 73, gene alterations and expression in primary oral and laryngeal squamous carcinomas. Carcinogenesis. 2001, 22:729-35.

23. Yoo GH, Washington J, Oliver J, Piechocki M, Kim H, Nora JF, Shibuya TY, Wilson DR, Ensley JF. The effects of exogenous $\mathrm{p} 53$ overexpression on hpv-immortalized and carcinogen transformed oral keratinocytes. Am Cancer Soc. 2002, 94:159-66.

24. Rouband-Diogene H, Fortin A, Morency R, Roy J, Monteil RA, Têtre B. Markers of radioresistance in squamous cell carcinoma of the head and neck: a clinicopathologic and imunohistochemical study. J Clin Oncol. 1997, 8:1030-8. 
25. Couture C, Diogéne HR, Têtu B, Bairati I, Mury D, Allard J, Fortin A. p53 and ki-67 as markers of radioresistance in head and neck carcinoma. Am Cancer Soc. 2002, 94:71322.

26. Gluckman JL, Pavelic ZP, Welkoborsky HJ, Mann W, Stambrook P, Gleich L, Wilson K, Righi P, Portugal LG, Mcdonald J, Biddinger, Steward D, Gartside P. Prognostic Indicators for Squamous Cell Carcinoma of the Oral Cavity: A Clinicopathologic Correlation. Laryngoscope. 1997, 107:1239-1244.

27. Brachman DG, Beckett M, Graves D, Haraf D, Vokes E, Weichselbaum RR. p53 mutation does not correlate with radiosensitivity in 24 head and neck cancer cell lines. Cancer Res. 1993, 53:3667-9.

28. Pirollo KF, Hao Z, Rait Yang YJ, Fee Wee JR, Ryan P, et al. p 53 mediated sensitization of squamous carcinoma of the head and neck to radiotherapy. Oncogene. 1997, 14:1735-46.
29. Lavertu P, Adelstein DJ, Myles J, Secic M. p53 and ki67 as outcome predictors for advanced squamous cell cancers of head and neck treated with chemoradiotherapy. Laryngoscope. 2001, 11:1878-92.

30. Myers JN, Elkins T, Roberts D, Byers RM. Squamous cell carcinoma of the tongue in young adults: increasing incidence and factors that predict treatment outcomes. Otorhinolaryngol Head Neck Surg. 2000, 122:44-51.

31. Weichselbaum RR, Kufe DW, Advani SJ, Roizman B. Molecular targeting of gene therapy and radiotherapy. Acta Oncol. 2001, 40:735-8.

32. Goepfert H. Squamous cell carcinoma of the head and neck: past progress and future promise. CA Cancer J Clin. 1998, 48:195-198.

33. Hamilton PW, Allen DC. Morfometry in histopathology. Journal of Pathology. 1995, 175:369-79. 